\title{
Strates
}

STRATES Matériaux pour la recherche en sciences sociales

8 | 1995

La question de l'environnement: recherches parallèles en Espagne et en France

\section{Doñana : tradition et changement social}

Paradoxes d'un espace naturel charismatique

Juan Carlos González Faraco

\section{(2) OpenEdition}

\section{Journals}

Édition électronique

URL : http://journals.openedition.org/strates/887

DOI : $10.4000 /$ strates. 887

ISSN : $1777-5442$

Éditeur

Laboratoire Ladyss

Édition imprimée

Date de publication : 30 juin 1995

ISSN : 0768-8067

\section{Référence électronique}

Juan Carlos González Faraco, « Doñana : tradition et changement social », Strates [En ligne], 8 | 1995,

mis en ligne le 20 décembre 2005, consulté le 08 septembre 2020. URL : http://

journals.openedition.org/strates/887 ; DOI : https://doi.org/10.4000/strates.887

Ce document a été généré automatiquement le 8 septembre 2020.

Tous droits réservés 


\section{Doñana : tradition et changement social}

Paradoxes d'un espace naturel charismatique

Juan Carlos González Faraco

\section{NOTE DE L'ÉDITEUR}

Traduit de l'espagnol par Yves Luginbühl. (Ce travail est issu de l'intervention de l'auteur dans un cours sur Doñana dirigé par le Dr. Javier Castroviejo, qui s'est tenu en août 1992 à l'Université Hispano-américaine de La Rábida (Palos, Huelva) ${ }^{1}$

«...au milieu de cette sauvage et éclatante

nature ».

Antoine de Latour, 1858.

«Las lagunas de Almonte, entre fresnedas y alamedas, son tantas como las arenitas de la

mar».

Camilo J. Cela, 1961.

Dynamisme social et conflit dans la zone de Doñana

1 Le texte qui suit, empreint de surprise et d'espoir, constitue l'annonce, par la mairie d'Almonte ${ }^{2}$, de l'apparition dans son territoire municipal d'une zone protégée, réserve scientifique et amorce sans doute de l'actuel Parc National de Doñana :

"Le Conseil Supérieur de la Recherche Scientifique a acquis récemment, afin de constituer une station biologique, une partie de la célèbre réserve de chasse de Doñana, limitrophe de la plage; son avifaune unique au monde constituera un motif d'attraction touristique en raison de la présence d'oiseaux migrateurs rares et divers, derniers survivants d'espèces du continent européen, auxquels s'ajoute un abondant gibier de grande taille. Il est surprenant que ce contexte de conditions favorables n'ait pas été jusqu'ici exploité pour inciter au développement de ce littoral $» .^{3}$ 
C'était en 1965 et la municipalité prenait conscience de l'événement avec un grand retard - la réserve avait été créée l'année précédente. Elle mettait de grands espoirs dans un développement touristique qui avait été annoncé depuis 1962 avec les premières intentions de planification urbaine sur le littoral - c'est-à-dire le futur Matalascañas. Le secrétaire de mairie rédacteur de son rapport annuel ne se doutait pas que débutait également un parcours hérissé de malentendus, de polémiques et de conflits. D'un côté, ceux qui s'affirmaient dans le champ de la protection de la nature, de l'autre ceux qui se consacraient à la promotion ou à l'exploitation économique d'un littoral jusqu'alors vierge; avec cette curiosité supplémentaire que l'on trouvait quelquefois la même institution, en l'occurrence l'État, dans les deux camps".

Protéger ou développer: ce sera le dilemme et le sujet d'une histoire chargée de tensions. Doñana, jusqu'à ces dernières années, avait représenté pour les communes limitrophes un espace éloigné et stérile, fréquenté épisodiquement par une faible population d'éleveurs, de vivanderos ${ }^{5}$, de pêcheurs et de cueilleurs des mille et une ressources qu'offrait la terre. Terre ingrate, isolée du monde par des rias continues et vastes, bourbier où régnait le paludisme et où vivre était seulement accessible à quelques clans familiaux qui y étaient nés et y avaient été élevés. Terre oubliée par les agronomes et la bureaucratie ${ }^{6}$. La prétention des conseils municipaux d'Almonte à l'époque de la IIe République (1931 et 1936), qui consistait à exproprier ces sables à des fins de réforme agraire, fut l'une des rares occasions où l'administration locale reconnut à Doñana un éventuel intérêt économique, davantage d'ailleurs pour des raisons idéologiques ou par idéal que par pragmatisme.

En 1965, avec l'ouverture de la route entre El Rocío et l'urbanisation projetée - loin de l'ancien «chemin de la mer» qui unissait Almonte aux plages -, avec l'approbation récente du projet de promotion touristique du littoral de Huelva, avec la déclaration également récente du pèlerinage du Rocío comme «Fête d'intérêt touristique » et avec le début des travaux d'aménagement du littoral (1966), la conversion de l'« ancienne réserve » en " paradis pour la science » était ainsi un événement nouveau qui s'ajoutait aux autres. Il orientait le regard admiratif de ces populations vers un Sud oublié, ou tout au moins distant de leur vie quotidienne ancrée depuis des siècles dans un espace proche de leurs habitations.

6 La présence du parc, créé par décret en 1969, ne sera pas ressentie pendant les années immédiatement postérieures comme une entrave. En définitive, les obstacles qui s'opposaient à la fréquentation de cet espace et à l'usage de ses ressources naturelles n'étaient pas nouveaux: la propriété et le contrôle restaient dans les mêmes mains. Entre 1971 et 1976, la mairie d'Almonte s'accommoda de la présence du parc en l'embellissant de projets de développement - une route littorale et un lycée -, alors même que survenaient les premiers heurts et les premières divergences entre les protectionnistes et le pouvoir local. En 1976, la mairie d'Almonte tenta, par exemple, de contester les limites du parc dans sa partie littorale. Mais c'est à partir de 1979, avec la requalification et l'extension du parc, tandis que s'établit la démocratie ${ }^{7}$, que s'accroît l'animosité et que les divergences deviennent une véritable hostilité envers ce que «symbolise» le parc: un fait écologique introduit par des étrangers, qui impose des contraintes et qui freine le développement de ces communautés.

7 En réalité, ce n'est pas l'unique paradoxe que Doñana a produit dans son histoire où le mythe - depuis Tartessos, peut-être - se confond avec la réalité, où les images surpassent de beaucoup les faits. Ses sols eux-mêmes étaient marqués par le contraste brutal : le 
sable et l'argile, le sec et l'humide. "Le sable, pour les perdrix; je suis fils de l'eau ", disait avec esprit un ancien marismeño ${ }^{8}$ qui délimitait fort bien les deux grands espaces, aujourd'hui unis par le même nom et auparavant séparés par la géographie et les deux modes très différents de vie.

8 Sable et argile signifièrent, en outre, deux modes de rapport très différents de ces communautés à leur milieu. Le sable, couvert de forêts et de maquis, trouva rapidement - vers 1300 - un régime de propriété seigneuriale et ses limites furent vite parfaitement fixées. La chasse, dans ces fourrés épais, constituait la plus ancienne et habituelle activité. Elle donna lieu à deux figures distinctes en conflit permanent: le chasseur aristocrate qui tirait les marcassins, abattait les cerfs et s'entourait de gardiens chargés de la surveillance de sa propriété (Ojeda Rivera, 1987), et le braconnier. Après l'interdiction de cette activité pour des motifs de protection, en désaccord avec les propriétaires héritiers de cette tradition cynégétique, le conflit subsiste, même avec d'autres arguments. La poursuite du braconnier obéit aujourd'hui à des motivations écologiques. Et ce qui se faisait au nom des privilèges d'une oligarchie se fait aujourd'hui au nom du droit cautionné par les lois de la majorité. La « réserve » est désormais "parc»: terra omnium que les habitants des environs continuent à voir comme la terre de quelques-uns (scientifiques, écologistes, protectionnistes, politiques), aristocratie éclairée qui n'est pas distincte, qualitativement, de celle qui auparavant représentait les anciens seigneurs.

Quant au marais, terra nullius, affecté pendant très longtemps par un régime de propriété moins rigide et plus ouvert - municipal et/ou sectionnal -, il fut un territoire plus accessible et ses ressources plus facilement utilisables, en particulier les prairies, pratiquées par un élevage extensif, accueillant aujourd'hui vaches et juments. Actuellement, cette double image s'est convertie en une seule sous l'effet de la juridiction protectionniste, dans une aire que l'homme avide a réduite jour après jour. Le sens de la limite et de la réserve, teinté de l'étouffante sensation d'un lieu assiégé par les cultures et les machines, autrefois seul domaine des sables couverts de frondaisons, a atteint également les zones inondables qui survivaient aussi à l'état naturel. Sa signification s'est également altérée peu à peu. De ressource pour des activités primaires en rien technicisées, alternative et complément pour un groupe important de propriétaires agriculteurs - à Almonte, pelentrines -, cet espace est devenu symbole, marque d'identité, motif de poèmes et de fêtes, à l'origine d'un sociocentrisme (Caro Baroja, 1957) croissant.

10 Aujourd'hui plus que jamais, cette optique locale, fondée sur des critères comme la tradition ou l'autochtonie, s'oppose à celle pour laquelle le parc, emblème de Nature, représente un fait et un symbole charismatique universel. L'émergence des valeurs de ce territoire sur la scène internationale avec, en 1960, la publication du livre de José Antonio Valverde sur les vertébrés de Doñana marque peut-être le début de ce paradoxe qui, avec le temps, est devenu intenable. Il convient cependant de rappeler le précédent introduit par Abel Chapman qui plaçait Doñana dans le Guide mondial de l'exotisme dès le début de ce siècle (1910). Mais ce sont Valverde et ses proches, déterminés, qui donneront une épaisseur scientifique aux ressources naturelles qu'abritent ces terres et convaincront la toute récente World Wildlife Fund (WWF), créée en 1961, de la nécessité de la protection. Aujourd'hui Doñana, Réserve de la Biosphère, dotée de mille et un titres, sujet de discussion dans les instances politiques 
internationales, objet d'étude pour des gens d'ici et là, a acquis le label de bien universel.

11 A cet ensemble d'oppositions symboliques, difficiles à synthétiser parce qu'elles se fondent tant sur des faits que sur des perceptions culturelles, et quelquefois sur des intérêts opposés, s'en ajoutent d'autres. La définition territoriale de Doñana elle-même est sujette à discussion. Il n'est pas besoin de remonter à la dimension écologique d'origine avant les premières transformations de grande ampleur, ni, non plus, à l'extension importante de la superficie protégée qu'entraîne la Loi promulguée à la fin de 1978. Actuellement, en outre, avec la récente déclaration de Parc Naturel périphérique de 1989, avec les délimitations spatiales que consignait le Plan Directeur Territorial (PDTC) de 1988, et celles qu'implique ensuite pour des objectifs de «développement durable» le rapport d'experts internationaux de 1992, le terme de Doñana désigne un espace en constante redéfinition. Étonnante évolution: depuis le très ancien toponyme d'un domaine de sable, quand la propriété appartenait aux ducs de Medina Sidonia, jusqu'au terme désignant un très vaste espace dans le triangle formé par la rive droite du Guadalquivir et le littoral atlantique et qui va d'ailleurs audelà du fleuve près de Sanlúcar de Barrameda. Un toponyme «énergique " qui a une capacité de séduction extraordinaire, comme c'est le cas pour El Rocío, qui est contigu : l'évocation d'une image de la Vierge finit par être le nom du hameau qui s'est développé dans son ombre9.

Ainsi, comme corollaire de cette ambiguïté "manichéenne ", nous nous trouvons en présence de groupes divers. Les uns, ayant l'esprit pragmatique des administrateurs, considèrent avec une précision géométrique les limites de la zone objet de leur gestion et de leur contrôle. De la même manière, mais avec des souhaits d'intervention économique, d'autres estiment trop vaste l'espace protégé, mais désirent voir au moins ses limites fixées et définitives. D'autres encore, dans une perspective écosystémique globale, interprètent ces limites avec une certaine souplesse scientifique, d'autres enfin, avec une pareille perspective, envisagent cet espace comme un ensemble varié et une région complexe: système naturel, socioculturel et économique, d'influence locale/régionale (Martinez Veiga, 1978) et sujet à des interactions de tout type (interne/internes, interne/externes, etc.) (Colom, 1979).

Cette ambiguïté éclaire les conflits qui opposent quelques localités proches à propos de la prééminence de telle ou telle municipalité dans l'usage des "produits " dérivés du $\operatorname{Parc}^{10}$. Ainsi la répartition administrative classique et parfois obsolète et arbitraire du territoire en communes resurgit et prend de la vigueur, quand on se trouve à la marge de formations politiques et territoriales transnationales engagées dans des conventions internationales, et au milieu d'un «village global » où la crise environnementale dépasse les frontières et où les distances paraissent se réduire de manière vertigineuse. Autre paradoxe à ajouter à ce scénario complexe que représente Doñana.

C'est jusqu'aux idées sur la tradition et la modernité qui dérogent à leur définition et deviennent des recours avec une étonnante légèreté dans les critères utilisés. Dans les brochures et les expositions, on a qualifié Doñana de "paysage changeant », et en effet il l'a été et continuera de l'être. Mais de la même manière nous aurions pu le qualifier d'espace culturel et social dynamique. Il reste peu de ce « désert humain " qu'Antoine de Latour parcourut dans son voyage au Rocío au milieu du XIXe siècle (1858). Il reste peu de ce pèlerinage de vingt-quatre heures dont l'unique rituel distinctif consistait dans une procession de l'Image Vénérée au milieu de la matinée du lundi de Pentecôte, à un 
rythme tranquille, dans l'« immense prairie » qui entourait l'ermitage. Il reste peu de cette brève réunion de quelques milliers de pèlerins ${ }^{11}$ (Murphy, 1989, tableau I) qui chaque année se retrouvaient dans un chemin pénible à travers des terres "dépeuplées d'hommes et peuplées de fauves $»^{12}$.

Les traditions et leurs changements se succèdent, leur coexistence et leur délimitation sont parfois difficiles, surtout à partir de ces dernières décennies où un train de nouveautés est apparu dans ces terres.

Mais les images, les symboles, les rites, contiennent plus de force que les faits et pour cette raison, le conflit et l'opposition sont les formes les plus constantes de la perception par l'homme de Doñana et son monde. Pour illustrer cet état instable, inachevé, rétif à toute définition, nous utiliserons trois exemples très significatifs: d'abord, El Rocío, l'un des centres habités qui reproduit le mieux dans ses fondements les contrastes et les changements intenses qui ont eu lieu dans la région; comme deuxième exemple, les pratiques d'élevage dans les marais; enfin, comme troisième illustration, dans le cadre d'une recherche en cours à caractère socio-éducatif, avec une orientation plus prospective que rétrospective, nous analyserons les représentations que les jeunes générations des environs se sont faites et se font de Doñana. Malgré leur singularité, ce parc national et les phénomènes qui lui sont associés produisent des paradigmes et leur analyse peut être étendue à bien d'autres espaces sociaux et/ou naturels de notre environnement culturel.

El Rocío : analyse de son évolution urbaine et démographique

El Rocío, hameau de la commune d'Almonte, situé au bord même des marais de Doñana, incarne mieux que tout autre lieu habité des environs du parc les différents modes du changement culturel et social qui a eu lieu dans cette région. Nous disposons de quelques descriptions de visiteurs étrangers du XIX ${ }^{e}$ siècle - dont celle, déjà citée, du Français Antoine de Latour - et des propres habitants du village ${ }^{13}$, grâce auxquelles il est possible de reconstituer le genre de vie, la démographie et l'organisation urbaine de ce « lieu sacré ». Le document municipal intitulé Chaumières du Rocío et ses habitants fait état, vers le milieu du XIX ${ }^{e}$ siècle, de trente-quatre constructions en matériaux naturels (pisé, jonc, genévrier de Phénicie, pin), avec une seule exception, celle de l'ermitage et de la maison annexe du moine. Toutes se concentraient irrégulièrement autour de « El Real » - espace plat voisin de l'église - et étaient généralement inhabitées. Il semble également qu'à proximité du chemin de Moguer, à quelques centaines de mètres du Real, existaient deux autres hameaux comptant une population plus ou moins stable : El Hato del Rincón et la Venta de los Colonos (Ojeda, op.cit.). Dans l'ensemble, une douzaine de familles avaient dans ce lieu leur résidence habituelle, sans autre activité que le pastoralisme, la chasse ou la cueillette et une agriculture limitée sur les friches voisines.

Cette situation se modifiera lentement, sous l'effet parcimonieux des cycles naturels. La première date notable est 1919 , année de la célébration solennelle du couronnement de la Vierge. A cet événement assistent, selon les récits de l'époque, environ vingt-cinq mille personnes et la première automobile : un présage de ce qui allait advenir. Entre 1925 et 1935, le Conseil municipal d'Almonte concède 92 droits à bâtir pour édifier de nouvelles constructions, en plus des 40 autorisées durant les cent vingt-cinq années antérieures. En 1927, le «Conseil général » de Huelva accorde la construction d'un chemin entre Almonte et El Rocío. Entre 1925 et 1930, le hameau voit installer son premier téléphone qui en sera l'unique jusqu'à une époque récente. En 1928, on 
aménage l'ancien chemin de Los Puertos pour son éventuel usage par les automobiles. En 1929 apparaît la première affiche annonçant le pèlerinage. A partir de 1931, la corporation républicaine tente d'engager et d'ordonner la croissance urbaine du hameau, en facilitant les concessions et la normalisation des terrains à bâtir et en lançant une timide campagne publicitaire. En 1958 la route Almonte-El Rocío est tracée et un pas est ainsi franchi vers un processus de colonisation qui, en synergie avec l'urbanisation littorale qui se développe immédiatement après, complète une double offre touristique. C'est pratiquement dans ces termes que le relate le Mémoire du premier plan urbain du Rocío de février 1970, bien que le rapport antérieur de la Commission provinciale d'urbanisme formule expressément l'objectif visant à éviter «la transformation du hameau en un village permanent ou en village de villégiature » (ibidem). Les faits contrastent avec ce souhait et bientôt commencent - avec un essor grandissant - les procédures d'adjudication publique de parcelles. Les constructions iront en se développant, comme le commerce de location de maisons pour le pèlerinage, jusqu'à fournir du hameau, dans les décennies postérieures, l'image d'un espace étendu et quadrillé, où les critères d'urbanisme géométrique s'unissent à ceux d'une esthétique andalouse conventionnelle éloignée des maisons austères d'Almonte et plus encore des habitations traditionnelles du Rocío.

Il est intéressant de noter que dans le même temps où se développe la fréquentation touristique et où s'encourage la prolifération de maisons à des fins festives ou de séjours de vacances, sans la moindre référence ni considération pour les habitants habituels du village, les responsables locaux réitèrent leur attachement à «la défense du caractère du village ": ils s'opposent par exemple à l'édification à proximité d'un hameau destiné aux colons du Plan d'irrigation Almonte-Marais; ils se réjouissent encore d'offrir, en fournées successives, des terrains à la Mère des Marais, afin d'étendre le périmètre voisin de l'ermitage ${ }^{14}$. Encore une fois la tradition s'accommode des nécessités du progrès : les deux termes sont interchangeables et leurs significations respectives se diluent rapidement.

Cependant, l'image du village comme lieu exotique, presque dépeuplé, et expression définitive du naturel, prospère. Dans un article de presse (Diario 16, 13/5/89), à l'occasion du pèlerinage de 1989, le journaliste, pour estimer la population du village, affirme qu'elle ne dépasse pas la centaine d'habitants... (cf. tableau 1). Les autorités locales elles-mêmes s'étonnent quand, au début des années quatre-vingt, les maîtres d'école ${ }^{15}$ insistent sur la nécessité d'ouvrir de nouvelles classes parce que la population scolaire a dépassé les deux cents élèves. C'est pourquoi il n'est pas surprenant que les habitants du Rocío soient depuis un certain temps désabusés du fait de l'oubli social dont ils souffrent, des déficiences de services et d'équipements dans le village, et répètent qu'ils se sentent marginalisés au point de plus même pouvoir s'appeler « Rocieros » : un terme tant à la mode qu'il a été approprié par les gens "d'ailleurs » ${ }^{16}$.

21 Finalement, le hameau s'est converti en bourg, mais aussi en centre de pèlerinage ; c'est un site de vacances et de loisirs de fin de semaine, montrant avec clarté le caractère indéfini de tout espace humanisé sur lequel pleuvent des interventions externes, diverses et simultanées.

Des 1744 habitations actuelles - selon le registre des contribuables d'Almonte de mars 1990 -, seulement 471 , c'est-à-dire $27 \%$, sont occupées toute l'année par des habitants du village ; $37 \%$ appartiennent à des résidents d'Almonte; $14,6 \%$ à des habitants d'agglomérations proches - Séville et Huelva ; $17 \%$ à des habitants des provinces de ces 
villes et en particulier à des personnes domiciliées dans des villages proches du Condado de Huelva ou de l'Aljarafe sévillan, et le reste à des personnes d'autres provinces andalouses ou d'autres Communautés autonomes. L'espace urbanisé prévu par les plans spéciaux successifs est en grande partie occupé et le prix du terrain a ainsi augmenté jusqu'à des extrêmes insoupçonnables. Lointaines sont aujourd'hui, davantage dans la mémoire que dans le temps - nous parlons des années soixante -, les considérations de la mairie sur "l'improductivité de ces terres» justifiant leur " inutilité publique ", ou les décisions pittoresques comme l'autorisation de pâturage dans des lieux aujourd'hui transformés en rues grouillantes, ou la récolte de bayunco là où campent désormais des milliers de pèlerins. Les villageois de nos jours se répartissent dans les zones périphériques, dans le fameux «quartier des Gallinas », de l'autre côté de la route, aux bords du chemin de Moguer. Un autre secteur important est constitué par la populaire rue Ajolí, dans la dernière zone d'expansion du Rocío. Ces deux noyaux de population sont éloignés l'un de l'autre et des signes de développement de bidonvilles sont apparus, comme un symptôme peu souhaitable de future banlieue.

Peut-être ce processus mérite-t-il d'être étudié avec plus de précision. Nous utiliserons ainsi les chiffres des recensements de population de la commune d'Almonte entre 1930 et 1989. Tous les phénomènes qui sont apparus dans cette zone (socialisation des rites du Rocío, «boom » touristique, amélioration des communications, élévation du niveau de vie, processus de changement culturel et formes de vie généralisées dans tout le pays, avec ses significations particulières en Andalousie, plus un grand et coetera de facteurs) ont entraîné, avec un impact différent mais en interaction continuelle, un brusque changement de chaque indicateur sociologique que nous allons examiner.

Tableau 1 : Évolution démographique de El Rocío (1930-1989)

\begin{tabular}{|l|l|l|l|l|l|}
\hline & $\mathbf{1 9 3 0}$ & $\mathbf{1 9 5 0}$ & $\mathbf{1 9 7 0}$ & $\mathbf{1 9 8 0}$ & $\mathbf{1 9 8 9}$ \\
\hline \hline Population & 199 & 258 & 553 & 774 & 1011 \\
Familles & 49 & 74 & 127 & 179 & 255 \\
Personnes/famille & 4,0 & 3,5 & 4,3 & 4,3 & 3,9 \\
Nés à Almonte (\%) & 97,0 & 80,6 & 77,8 & 61,3 & 78,0 \\
Régions d'origine & 3 & 2 & 43 & 51 & 62 \\
\hline
\end{tabular}

Source : Recensement des habitants, Mairie d'Almonte, élaboration de l'auteur.

Il convient tout d'abord de préciser que ces données ${ }^{17}$. proviennent d'une étude du répertoire des rues du village d'où nous avons éliminé les individus ou ménages résidant dans des zones proches (Doñana par exemple) que les agents recenseurs ont inclus dans le recensement du Rocío. La première observation, qui ne présente aucun caractère exceptionnel, révèle un accroissement de population important, à un rythme plus rapide à partir des années soixante. Les données officieuses que nous possédons aujourd'hui montrent que cet accroissement n'a pas cessé, ce qui provoque une augmentation de la proportion d'individus par habitation grâce à la construction d'étages supérieurs, la séparation des maisons familiales en plusieurs unités ou leur extension dans les cours annexes, en raison des énormes difficultés financières des villageois pour construire de nouvelles habitations dans d'autres parcelles ${ }^{18}$. Dans la 
période de 1930 à 1970, la population a quasiment triplé. Par rapport à la première date, la population actuelle est cinq fois plus élevée, alors que dans l'ensemble du territoire communal l'augmentation enregistrée a été seulement de deux et demi au cours du siècle. Cette estimation pourrait en outre présenter un certain taux d'erreur et devrait être corrigée à la hausse si l'on considère que la population de fait est avec certitude supérieure à la population de droit.

La même observation doit être faite avec le nombre de familles, bien que la proportion de personnes par famille soit à peu près constante, avec une légère tendance à la baisse. La diminution de la natalité est plus claire, comme celle du nombre d'enfants par famille. Le fait le plus frappant, en tout cas, est constitué par l'accroissement spectaculaire du nombre de communautés d'origine. Actuellement, les natifs du Rocío ou d'Almonte, tout en étant majoritaires, se partagent le village avec pratiquement $25 \%$ de personnes nées dans 61 communautés distinctes. C'est dire si l'hétérogénéité des Rocieros, quant à leur origine, est élevée : de 3 communautés en 1930 à 62 à la fin des années quatre-vingt. On ne peut que constater une irrésistible tendance à la complexité sociale, convergeant avec celle que l'on peut observer dans son environnement démographique et signe évident de perte de l'isolement et de diversité croissante.

Tableau 2 : Population active et secteurs d'activité. El Rocío (1930-1989).

\begin{tabular}{|l|l|l|l|l|l|}
\hline & $\mathbf{1 9 3 0}$ & $\mathbf{1 9 5 0}$ & $\mathbf{1 9 7 0}$ & $\mathbf{1 9 8 0}$ & $\mathbf{1 9 8 9}$ \\
\hline \hline Pop. active (nbre absolu) & 80 & 95 & 184 & 203 & 323 \\
Pop. active (\%) & 40,2 & 36,6 & 33,3 & 26,2 & 31,9 \\
Pop. act. fém./pop. act. & 1,2 & 3,2 & 1,6 & 12,3 & 18,9 \\
Secteurs d'activité (\%) & - & - & - & - & - \\
- Primaire & 93,7 & 80,0 & 77,7 & 52,7 & 50,5 \\
- Secondaire & - & - & - & - & 0,6 \\
- Tertiaire & 6,3 & 20,0 & 22,3 & 47,3 & 48,9 \\
Types d'activités & 3 & 8 & 19 & 30 & 36 \\
\hline
\end{tabular}

Source : Recensement de la population. Mairie d'Almonte, élaboration de l'auteur.

Le tableau 2 révèle une lente chute, avec quelques aspérités toutefois, de la population active, peut-être due à l'accroissement de la durée moyenne de scolarisation des jeunes, à leur plus tardive insertion dans le marché du travail et à la stabilisation de l'âge de la retraite: un luxe pour les Rocieros des années trente. L'augmentation, faible mais visible, de la population active dans la décennie quatre-vingt peut s'expliquer par l'un des processus de changement et de modernisation sociaux du village: l'accès des femmes au monde du travail. Depuis un pourcentage insignifiant en 1930 (1,2\%), les femmes ont atteint une présence significative dans le travail : pratiquement $19 \%$ des actifs sont des femmes.

Dans la distribution de la population active par secteurs d'activité, nous pouvons noter quelques faits importants: la diminution continue et intense du secteur agraire (cultivateurs, éleveurs, etc.) et sa diversification interne. En effet, en 1930, la presque 
totalité des actifs appartenaient à ce secteur - sauf un santero ${ }^{19}$ - qui était identifié par deux indicateurs uniques: "champs» et «élevage». En 1989, le panorama est diamétralement opposé : 50,5\% de la population déclarent leur appartenance au secteur agricole en la nuançant très souvent par une spécialité. Ce chiffre peut d'ailleurs être considéré comme éloigné de la réalité, en raison de l'existence d'un groupe important d'individus pluri-actifs (entre 10 et $15 \%$ ) se partageant entre cette activité et la construction. On connaît bien cette tendance non négligeable à se déclarer membre de la branche agricole à des fins administratives - percevoir des indemnités du chômage, par exemple - bien que les revenus essentiels proviennent de la construction ou d'autres branches d'activité.

Il faut souligner également le poids relatif du secteur tertiaire, où les services publics, insuffisants mais à prendre en compte, s'ajoutent au secteur hôtelier ${ }^{20}$ et à la construction, ces deux activités représentant un volume de travail appréciable. La présence significative du secteur public illustre avec clarté l'insertion du Rocío dans le domaine d'intervention et de contrôle de l'État, fait si commun qu'il ne surprend que dans un cas comme celui-ci, où le village a été pendant si longtemps éloigné de la dynamique sociale de son environnement sociologique et politique.

D'autres données confirment ces extrêmes. En 1980, par exemple, on n'accordait aucune attention aux enfants d'âge pré-scolaire et la population infantile scolarisée s'entassait dans quatre salles - dont deux de plus de vingt-cinq ans d'ancienneté. D'après les chiffres de 1993, nous savons que El Rocío compte désormais dix-huit salles de classe auxquelles accède une population de plus de 300 élèves âgés de quatre à quinze ans. Très peu d'entre eux (entre 10 et $14 \%$ ) poursuivent leurs études. Malgré les améliorations indubitables du secteur éducatif, le rapide processus de changement social et l'élévation du niveau de vie ne correspondent pas à une qualification de la main-d'oeuvre ni à une élévation du niveau moyen d'éducation conforme à l'époque. Ce phénomène est général dans l'ensemble de la commune d'Almonte, où $18 \%$ des personnes âgées se déclarent analphabètes et 70 \% (données de 1989) sans diplôme, pratiquement illettrées selon les critères actuels. C'est pourquoi il est normal de constater que la majorité de la population active se rencontre dans les emplois les moins qualifiés et que sa fragilité et sa dépendance à l'égard de conjonctures économiques instables soient d'autres traits caractéristiques et préoccupants.

Cette série de données nous conduit à quelques conclusions plausibles: au cours des soixante années étudiées, les différences, autrefois radicales, entre une population résidant sur des terres sans utilité reconnue, homogène et anachronique, et son environnement géographique proche se sont effacées. Du paradigme de primitivisme avec des habitants offrant l'image stéréotypée de l'homme naturel, on est passé en deux décennies seulement à un village en évolution, avec des signes forts de changement social et des perspectives nettes de développement économique classique basé sur la rente du tourisme (festif, de loisir, de nature), sur les célébrations traditionnelles aujourd'hui socialisées et sur une nouvelle agriculture qui passe actuellement par des phases critiques.

Cependant le regard extérieur voit se renforcer son image de "nature», de "campagne", de lieu résumant quelques caractères remarquables de ce qui est habituellement considéré comme cliché de l'«identité andalouse ». Ainsi émerge sa beauté originelle - nous pensons à la riche série de poèmes populaires (Gonzalez Faraco, 1992) - qui s'unit à Doñana dans un ensemble symbolique commun. Ces impressions 
contrastent avec les données démographiques, avec celles de l'évolution urbaine, avec les nouveaux usages et rites sociaux et avec la perception que les habitants ont d'euxmêmes. La frontière entre El Rocío et les Marais voisins, autrefois subtile et sans heurt, se fait chaque jour plus évidente et se pare d'aspérités ${ }^{21}$.

Les juments des marais : transformations d'une tradition pastorale

Comme deuxième illustration des effets du changement social survenu dans l'aire de Doñana, nous avons choisi l'un des ces usages traditionnels qui subsistent encore et qui gagnent même en audience : l'élevage de chevaux sauvages dans les marais. Nous ne nous étendrons pas sur des considérations liminaires que nous avons déjà retracées en d'autres occasions (cf. bibliographie). Nous nous contenterons d'esquisser les processus de changement survenus dans cette activité et de réfléchir à leur signification actuelle, bien que leurs protagonistes recourent continuellement à la tradition et refusent de reconnaître les altérations notables qu'elle a subies.

Il semble que l'usage pastoral des marais se perde dans le temps. Sans mentionner les mythiques taureaux de Tartessos, on sait que les Arabes élevaient des chevaux et des juments de reproduction ${ }^{22}$. Plus tard s'y joindront les troupeaux transhumants liés à la législation avantageuse de la Mesta ${ }^{23}$. Et traditionnellement, les habitants des environs utilisaient les pâturages pour des troupeaux de porcs, de brebis, de vaches et de chevaux, animaux "sauvages et indomptés » qui divaguaient en toute liberté dans ces solitudes ${ }^{24}$. Aujourd'hui cette activité se poursuit, réduite pourtant aux vaches et aux chevaux, dans certaines enclaves des marais de Hinojos. Mais en général l'élevage, et pas seulement celui des marais, n'est plus qu'un secteur résiduel sans signification dans les économies de ces communes.

La présence du bétail a posé quelques problèmes aux gestionnaires et protecteurs de la nature, surtout en raison de la concurrence qui s'exerce avec les ongulés sauvages et herbivores, et la pénétration de personnes dans un territoire régi par des principes protectionnistes comme ceux d'un Parc National. Ces circonstances ont ajouté un nouveau conflit qui, cette fois, ne se pose pas en termes d'utilisation économique d'une ressource - tout du moins ce n'en est pas l'aspect primordial- mais en termes dialectiques de protection écologique et/ou de droit héréditaire ${ }^{25}$ (Ojeda, 1993, pp. 70-72).

Le groupe majoritaire d'éleveurs de chevaux - les yegüerizos ${ }^{26}$ - a vu se réduire les aires de pâturage et s'accroître le contrôle public des espaces qu'il considère comme un patrimoine de sa culture historique et jusqu'à un facteur d'identité populaire. La majorité de ces éleveurs viennent de la commune d'Almonte, bien qu'une grande partie des pâturages s'étendent dans des terrains de Doñana qui sont la propriété de la commune d'Hinojos. Ils s'acheminent vers ces terres pour surveiller et conduire les animaux et surtout pour les encercler et les rassembler dans la matinée du 26 juin, quelques jours avant la Fête de San Pedro, autrefois marché au bétail et aujourd'hui simple célébration ludique (Murphy et González Faraco, 1988). Ce moment crucial de l'élevage est connu sous l'expression de saca de las yeguas ${ }^{27}$. Le bétail est amené par les chemins jusqu'au village et dans plusieurs corrals, les propriétaires marquent les poulains de l'année et coupent les crinières des adultes (la tuza ${ }^{28}$ [la tonte]). C'est également le moment des contrats, des échanges, qui se réduisent et se limitent de plus en plus aux usagers des marais eux-mêmes ou à ceux qui souhaitent s'insérer dans ce monde où la passion des chevaux, de ce qui est relatif au Rocío ou aux marais s'inscrit dans un unique modèle culturel. 
Les données dont nous disposons - discutables en raison des inexactitudes des comptages saisonniers - nous font penser qu'un cheptel d'environ 1300 bêtes très métissées paissent à l'intérieur du parc, dont $75 \%$ sont des juments. Les yegüerizos d'Almonte sont propriétaires de 800 à 900 têtes, mais la propriété se distribue de manière très particulière. La majorité ne possède pas plus de deux ou trois animaux à la différence de ce qui se passait dans les décennies antérieures: le nombre d'éleveurs était inférieur et la proportion de juments par propriétaire plus forte, de telle manière que les opérations de rassemblement et de conduite étaient beaucoup plus souvent confiées à des cavaliers salariés et experts dans ces entreprises. Avec de tels chiffres, il est difficile d'imaginer une quelconque rentabilité économique dans ce singulier mode d'élevage extensif. C'est pourquoi les yegüerizos l'ont peu à peu transformé en une activité où le caractère festif s'est lié au rituel, où le culturel s'est substitué à l'économique, le redéfinissant en une tradition communautaire, ainsi moins vulnérable aux interventions extérieures ou moins exposée à la disparition (Murphy, 1987).

Comme pour le village du Rocío et ses changements, nous pouvons discerner dans ce cas des explications sociologiques vraisemblables. Actuellement, l'élevage des chevaux repose sur l'émergence d'une nouvelle génération - d'une moyenne d'âge de 35 ans étrangère en grande partie au monde des éleveurs-agriculteurs qui gérait ce secteur auparavant. L'association privée ${ }^{29}$ qui s'est créée en 1982 pour le défendre et l'organiser est dirigée aujourd'hui par ce groupe de «nouveaux marismeños », issus des secteurs les plus divers de l'économie locale. Ils ont tourné leur regard vers le cheval en le considérant non comme une ressource économique et encore moins comme un instrument pour les travaux des champs, mais comme une passion ${ }^{30}$, une marque d'identité culturelle, qui renforce une idiosyncrasie locale, en partie enracinée, en partie inventée, en pleine transition et en plein changement. Avec des moyens modernes ils accèdent aux marais, réalisent les travaux nécessaires, profitent de moments de congés, récupèrent les vieilles sensations de l'homme perdu dans ces immensités, et pendant la saca de las yeguas ils s'habillent de vêtements traditionnels devant une multitude de spectateurs qui, de loin, sont les témoins de ce rite annuel et répété de temps à autre. Et tout ceci au coeur d'un magnifique morceau de nature, relique de ce qui fut des marais étendus de part et d'autre du fleuve.

La signification de Doñana dans la socialisation des nouvelles générations des environs

Nous ne ferons ici ni prophétie ni prospective raisonnée. Grâce aux données obtenues à partir d'une large enquête ${ }^{31}$ réalisée pendant l'automne 1991, nous allons simplement cerner la perception de Doñana par des groupes d'âge qui, dans les années futures, constitueront le groupe social principal de ces communautés des environs de Doñana. Ils vivent une situation de transition où règnent la controverse, les valeurs contraires, et où les heurts priment sur les rythmes réguliers. Dans un contexte où la relation singulière de ces populations avec les réserves de chasse et les marais a été rompue ou est en train de se dissoudre - elle était moins étendue et moins intensive qu'on ne le suppose aujourd'hui, où les anciens hommes des marais et les rares usagers de l'ancienne époque ont été convertis en images récurrentes et en symboles de la tradition collective -, les jeunes actuels se trouvent en situation d'affrontement avec des espaces déjà découverts et porteurs de valeurs ambiguës; ces espaces reconnus à l'échelle mondiale et objets de controverses dans leurs familles constituent, d'une part, un but plein d'illusions pour des visiteurs qui cherchent un de ces derniers refuges de 
la nature en Europe et, d'autre part, une terre et le cadre des pratiques fondatrices de l'affirmation locale.

39 Afin de quantifier ces hypothèses et d'éviter les critiques essentielles, nous avons effectué une enquête auprès de 300 élèves d'établissements d'enseignement de quelques localités des alentours de Doñana et d'âge compris entre 13 et 15 ans. Il s'agit d'élèves qui, proches de la fin de la période de huit années d'éducation élémentaire, ont eu l'opportunité de recevoir une formation générale indispensable pour des citadins d'une société moderne mais qui, malheureusement, n'ont que peu de possibilités ultérieures d'accéder à des connaissances académiques sur Doñana ou sur d'autres hauts lieux de l'environnement. Nous ne pourrons livrer que quelques conclusions provisoires et forcément générales, mais, d'une certaine manière, significatives. Nous disposons en outre de quelques informations supplémentaires qui peuvent compléter le contexte éducatif de ces groupes - portant en particulier sur des thèmes d'environnement - et offrir ainsi une idée des règles de base des processus de socialisation qui s'y déroulent dans le cours de leur dynamique socioculturelle.

Nous avions déjà connaissance de la faiblesse du niveau d'éducation sur l'environnement dans les établissements d'enseignement et du peu d'impact de l'administration, ainsi que de l'insuffisance des interventions éducatives informelles de caractère collectif après vingt-cinq années $d$ 'histoire de protection à Doñana. Les réponses des élèves confirment ces hypothèses. Environ $70 \%$ d'entre eux n'ont jamais participé dans leur vie scolaire à aucun des programmes éducatifs consacrés à Doñana réalisés de la part des instances concernées. Plus de $20 \%$ des élèves n'ont jamais visité le parc et seulement $40 \%$ ne l'ont fait qu'une seule fois, presque toujours avec l'école ou en famille. Il paraît encore plus alarmant que près de $22 \%$ de cette population scolarisée ne connaisse pas les installations annexes de réception des visiteurs.

Il arrive que l'on parle de Doñana dans les écoles, mais occasionnellement, avant une visite ou de manière anecdotique, et cependant les élèves estiment qu'il s'agit d'un thème auquel on ne donne pas l'importance qu'il mérite. Plus de la moitié des enquêtés pensent que les professeurs traitent ce thème peu ou presque jamais, bien qu'il représente pour eux un sujet intéressant et très motivant, autant que nous ayons pu en juger. En effet, la moitié des élèves estiment qu'il devrait inévitablement faire partie des programmes scolaires de cette région, l'autre moitié jugeant que cette inscription dans les programmes serait très opportune. L'intérêt des professeurs est en fait très divers et leur formation très insuffisante, de leur propre aveu.

Il apparaît ainsi que les premières idées et les connaissances sur Doñana cessent d'être, pour cette génération, un fait de transmission familiale. L'intensité des relations de ces populations avec Doñana s'est peu à peu affaiblie pour ce qui concerne les « usages traditionnels » - marginalisés par rapport à leur valorisation culturelle -, et ces mêmes relations se sont transformées depuis que le $\operatorname{Coto}^{32}$ s'est converti en un fait écologique et en un territoire institutionnalisé et régi par des lois. La moitié des élèves admettent avoir entendu parler pour la première fois de Doñana à l'école, dans les livres ou à la télévision, comme cela peut arriver à n'importe quel enfant de n'importe quel village éloigné. Ce qu'ils ont appris plus tard sur le parc provient, dans les trois quarts des cas, des écoles ou d'autres acteurs étrangers à leur milieu social ou à leur famille.

43 L'étude des connaissances de base sur Doñana (faune, flore, écosystèmes, processus et impacts écologiques, gestion administrative et recherche, usages traditionnels, actions contemporaines aux alentours, etc.) révèle des résultats consternants. Les analyses que 
nous avons réalisées auprès d'un échantillon représentatif ${ }^{33}$ montrent que l'instruction de $90 \%$ des élèves est très en dessous des « connaissances minimales » que l'on pourrait espérer pour une population qui effectue sa scolarité et vit à la porte d'un lieu aussi lié à sa communauté culturelle, d'une telle réputation et aussi fréquenté. Ces analyses révèlent des niveaux de connaissance qui ne vont pas au-delà des animaux les plus connus du parc et une déficience totale d'information sur les processus écologiques élémentaires des écosystèmes, sur la flore, les activités traditionnelles ${ }^{34}$ et bien évidemment sur les institutions gestionnaires et scientifiques qui agissent dans le parc. Il parait évident que les jeunes générations manquent du minimum d'information sur les composantes humaines et naturelles de leur milieu et sur les facteurs essentiels de son fonctionnement.

L'analyse des expressions graphiques et des propositions de thèmes de recherche qu'ont décrites spontanément les élèves confirme ces observations. Doñana est perçu comme un espace généralisé, stéréotypé, sans caractéristiques spécifiques particulières, interchangeable avec les autres espaces naturels, comme une globalité dont les parties intégrantes n'ont pas été analysées. L'image la plus commune est celle d'un territoire étendu, très réputé et plein d'animaux, avec la présence constante de l'eau, mais très peu différent de ce que n'importe quelle personne pourrait dessiner, sans apport d'autre connaissance que la rumeur ou la renommée du lieu. Les élèves ne remarquent pas les relations entre le parc et son environnement : la perception qu'ils en ont est celle d'un espace isolé sur lequel planent de grands dangers comme le braconnage ou d'autres agressions moins graves mais évidentes. La représentation de Doñana comme système régional, ou tout au moins comme idée générale est inexistante. Dans ces conditions on peut présumer de la difficulté de leur expliquer et de les convaincre des avantages des plans de développement qui les toucheront de plein fouet ${ }^{35}$.

45 En tout cas, leur attitude envers la nature est fondamentalement positive et leur niveau de motivation suffisant pour désirer l'étudier. Ils semblent cependant avoir assimilé quelques opinions locales qui peuvent être préoccupantes. En surévaluant l'importance de l'impact écologique du braconnage, ils attribuent une valeur certaine aux opérations technico-économiques en cours de réalisation ou projetées dans les environs du parc, bien que l'on sache qu'elles sont objectivement beaucoup plus dangereuses. Ils distinguent divers niveaux de fragilité ou d'utilité économique des écosystèmes. Et pour $60 \%$ d'entre eux, la plage n'est pas un paysage devant être protégé, pas plus que les pinèdes pour $18 \%$, ou les dunes pour $13 \%$. A leurs yeux, seuls les marais et les zones de maquis ont des caractéristiques d'écosystèmes totalement naturels. Tout ceci contraste avec un étonnant niveau de conscience sur les problèmes entraînés par le mauvais usage de l'eau : plus de $20 \%$ estiment que celui-ci est la cause des problèmes de Doñana, $17 \%$ les attribuent à une mauvaise information et $14 \%$ sont critiques à l'égard de l'administration du parc. Mais ils continuent à penser que ce sont les pratiques effectuées à l'intérieur du parc (chasse ou pêche) - pour 31 \% d'entre eux - qui sont les causes principales des problèmes.

Cette série d'observations et d'hypothèses incite à quelques conclusions claires: très bas niveau de connaissance de la réalité écologique du parc, perception peu analytique et déformée et système de valeurs ambigu ou tout au moins peu stable de Doñana et de sa signification. Si l'on tient compte des caractéristiques de la population étudiée (en fin de scolarité, proche de l'insertion dans le monde du travail) il ne nous paraît pas que 
les relations de Doñana avec son environnement et le désir affirmé d'une fonctionnalité de Doñana comme espace socio-culturel et naturel puissent s'appuyer sur une base solide. Il est, au contraire, plus raisonnable de voir se reproduire, à court terme tout au moins, la situation actuelle. Les mesures économiques ou de développement et l'engagement de certains programmes prévus peuvent contribuer à modifier cet état de choses, mais les processus de socialisation, à travers des valeurs fortement ancrées ou antagonistes, seront un obstacle permanent. S'il nous semble qu'une intervention pédagogique et/ou éducative est nécessaire, ses effets bénéfiques seront toujours tardifs et la spirale vertigineuse du processus social décrit n'aura jamais ni la patience d'attendre ni le sens de l'avenir que l'on pourrait souhaiter.

\section{BIBLIOGRAPHIE}

ALVAREZ GASTON, R. (1978), Almonte y El Rocío. Esperanzas de un pueblo andaluz, Sevilla, Editorial Católica Española.

CARO BAROJA, J. (1957), Razas, pueblos y linajes, Madrid, Revista de Occidente.

CARVALHO, J.C. \& MAGNANINI, A. (1982), « El papel de los Parques Nacionales y reservas similares en la civilización presente y futura ", in SIOLI, H. \& al. (1982) Ecología y protección de la naturaleza, Barcelone, Blume, pp. 414-441.

COLOM, A.J. (1979), Sociología de la Educación y Teoría General de Sístemas, Barcelone, Oikos-Tau.

COMELLES, J.M. (1991), « Los caminos del Rocío », in PRAT, J. \& al. (eds) (1991), Antropología de los Pueblos de España, Madrid, Taurus Universitaria, pp. 755-770.

COMISION INTERNACIONAL DE EXPERTOS (1992), Dictamen sobre estrategias para el desarollo socioeconómico sostenible del entorno de Doñana, Séville, Junta de Andalucia.

CHAPMAN, A. \& BUCK, W.J. (1910), Unexplored Spain, London, E. Arnold.

Coloquio Franco-Español sobre Espacios Naturales (1988), Madrid, Casa de Velázquez (3 vol).

GONZÁLEZ FARACO, J.C. (1991), «Efectos del cambio social en una práctica ganadera tradicional : la cría de caballos en las Marismas de Doñana », Agricultura y sociedad, 59, pp. 245-268.

GONZÁLEZ FARACO, J.C. (1991), « La evolución de los usos tradicionales en los espacios naturales protegidos ", Vida silvestre, 69, pp. 8-15.

GONZÁLEZ FARACO, J.C. (1991), « Evaluación del efecto educativo de los espacios naturales protegidos ", in I Jornadas de Educación Ambiental en Centros Educativos, Séville, Consejería de Educación y Consejería de Cultura y Medio Ambiente, pp. 49-52.

GONZÁLEZ FARACO, J.C. (1991), « Consecuencias sociales de la creación de un espacio protegido », Quercus, 69, pp. 22-25.

GONZÁLEZ FARACO, J.C. (1993), « La construcción lingüística del medio : Doñana en el floklore andaluz », II Jornadas de Enseñanza de la Lengua en Andalucía, Huelva, Aprela, pp. 91-96.. 
GRANADOS, M. (1989), Transformaciones históricas del Parque Nacional de Doñana, Thèse de doctorat, Université de Séville.

HERNANDEZ MONTESINOS, D. (1990), «Educación Ambiental en el medio rural : una estrategia de recuperación de la inteligencia social », Revista interuniversitaria de formación del profesorado, 7 , pp. 59-64.

LATOUR, A. de (1858), La Baie de Cadix. Nouvelles études sur l'Espagne, Paris, M. Lévy Frères.

MARTINEZ VEIGA, U. (1978), Antropología ecológica, La Coruña, Adara.

MATHIEU, N. et JOLLIVET, M. (dir.) (1989), Du rural à l'environnement, Paris, ARF/L'Harmattan.

MURPHY, M.D. (1987), « Marsh Mares of Almonte », The World \& I., 2 (5), pp. 452-469.

MURPHY, M.D. (1989), « Discovering Rocío : Rival Foundation Myths of an Andalusian

Pilgrimage ", Communication présentée à l'Annual Meeting of the American Anthroplogical Association, Washington D.C. (exemplaire mécanographié).

MURPHY, M.D. \& GONZÁLEZ FARACO, J.C. (1988), « Fiesta in Almonte », The World \& I, 3(7), pp. 480-491.

MURPHY, M.D. \& GONZÁLEZ FARACO, J.C. (1992), « La saca de la yeguas : the ritualization of an Andalusian horse roundup ", Communication présentée à l'Annual Meeting of the Southern Anthropological Society, St. Augustine, Floride (en cours de publication).

OJEDA RIVERA, J.F. (1985), « Le parc de Doñana et son environnement », Revue géographique des Pyrénées et du Sud-ouest, 56 (2), pp. 225-242.

OJEDA RIVERA, J.F. (1987), Organización del territorio en Doñana y en su entorno próximo (Almonte), Siglos XVIII-XX, Madrid, ICONA.

OJEDA RIVERA, J.F. (1993), Doñana : esperando a Godot, Séville, Instituto de Desarollo Regional, Cuaderno 31.

SUREDA, J. (1987), « Programas socioeducativos de Educación Ambiental no formal », in COLOM, A.J. y colaboradores (1987), Modelos de intervención socioeducativa, Madrid, Narcea, pp. 277-297.

VALVERDE, J.A. (1960), Vertebrados de las Marismas del Guadalquivir, Almeria, Instituto de Aclimatación.

WEST, P.C. \& BRECHIN, S.R. (eds.) (1991), Resident Peoples and National Parks. Social Dilemmas and Strategies in International Conservation, Tucson, The University of Arizona Press.

\section{NOTES}

1. Le texte original a été corrigé et actualisé pour cette édition. L'auteur le dédie aux éducateurs qui s'efforcent d'inculquer aux enfants et aux jeunes l'amour de la nature. Et tout particulièrement à la professeur María de los Angeles Garrido qui s'est vouée à ce rôle depuis des années dans l'une des communes appartenant au Parc National de Doñana.

2. Almonte est une commune voisine du Parc National de Doñana, située au sud-est de la province de Huelva. Une grande partie de son vaste territoire municipal est incluse dans le parc. Ses 17000 habitants (environ) se répartissent en trois noyaux de population : du nord au sud, Almonte, El Rocío et Matalascañas, en bordure des plages de l'Atlantique. 
3. Rapport annuel du secrétariat de mairie 1965, Mairie d'Almonte, Archives municipales, legs 304 , fol. 10.

4. Une telle accumulation de contradictions et son avenir incertain ne passèrent pas inaperçus pour ceux qui disposaient d'informations suffisantes et d'esprit critique. A la page 149 du fameux Rapport Foessa de 1975 on pouvait lire : « Dans les marais du Guadalquivir émergent actuellement des intérêts très divers et contradictoires. Le projet Guadalquivir-FAO, en cours d'exécution, prévoit l'assèchement à des fins agricoles de 120352 hectares, dont 38856 pour des cultures sèches, 49530 des cultures irriguées, 14400 des rizières et 17466 des sables pour l'irrigation. Simultanément, il est envisagé de maintenir un parc national et un centre de recherches biologiques, construire une route et urbaniser à des fins touristiques. Il existe également un projet proche de centrale nucléaire (Almonte). On peut apprécier l'urgence d'une planification engagée par les institutions administratives compétentes pour éviter un chaos qui entraînerait des pertes irréparables ». Estudios sociológicos sobre la situación social de España. (1976), Madrid, Fondation FOESSA et Editions Euramérica.

5. Les vivanderos ou marchands ambulants de tissus ou comestibles rendaient visite avec leur chevaux aux hameaux de Doñana et aux postes de carabiniers de la côte. Ainsi les nommait Alfonso Domínguez Domínguez, qui naquit et passa sa jeunesse à Doñana, dans un manuscrit de 1971 intitulé Notions d'Histoire et Mystère de la fameuse réserve de Doñana.

6. De nombreuses terres méridionales du territoire d'Almonte, comprenant Doñana, n'apparaissent pas dans certains cadastres en raison de leur « improductivité » et de leur caractère « marginal » (Ojeda Rivera, 1987, p. 27). Un autre fait significatif : entre 1949 et 1961, par exemple, le terme Doñana n'est jamais cité lorsque le lieu est évoqué dans les nombreux Actes Capitulaires d'Almonte.

7. Le 3 avril 1979, quelques mois après la promulgation de la nouvelle Constitution espagnole, ont lieu les premières élections municipales depuis la II ${ }^{\mathrm{e}}$ République. A Almonte, six groupes s'affrontent, dont trois indépendants : Union Libre d'Almonte (288 voix), Groupement Démocratique Indépendant d'Almonte (586 voix) et Union du Peuple d'Almonte (521 voix) ; les deux premiers sont des groupes conservateurs, le dernier appartient à la gauche. Le Parti Socialiste Ouvrier Espagnol (PSOE) remporte les élections avec 2702 voix et obtient la majorité absolue dans la municipalité. A une distance assez grande se situent l'Union de Centre Démocratique (UDC) avec 1233 voix et le Parti Communiste d'Espagne (610 voix). L'abstention dépasse $29 \%$. Le tournant que ces résultats électoraux impliquaient dans l'orientation du pouvoir local n'a pas entraîné un changement substantiel dans l'attitude méfiante et souvent hostile du Conseil municipal à l'égard des gestionnaires et des mesures protectionnistes du Parc National de Doñana. Dans cette période, par exemple, lors d'une réunion du Conseil municipal, l'un des principaux responsables de la recherche et de la protection de Doñana fut déclaré « ennemi public » d'Almonte. Cette municipalité, au début des années quatre-vingt dix et dans le cadre d'une orientation politique identique, renouvela une déclaration similaire.

8. Nous avons recueilli cette métaphore lors d'un entretien avec le garde, déjà retraité, Don José Rodríguez Parada, dit « Pepe Clarita », originaire de Sanlúcar de Barrameda et membre de l'un de ces groupes familiaux les plus attachés aux marais. (N.D.T. : marismeño n'a volontairement pas été traduit; ce terme désigne l'individu qui pratique les marais ou y réside.) 
9. En effet, l'évocation originelle de « Nuestra Señora de las Rocinas », débitrice du toponyme du lieu où fut érigé le premier ermitage, se transforma en « Nuestra Señora del Rocío ». A ce moment, ce sera le lieu lui-même qui changera de nom et s'appellera, comme l'effigie, « El Rocío ».

10. Dans une période récente, la municipalité d'Almonte a opposé un recours contre le nouveau Plan Directeur d'Usage et de Gestion du Parc National de Doñana, à propos de certaines mesures de ce plan relatives aux accès des visiteurs à partir de Sanlúcar de Barrameda. Jusqu'à maintenant, il existait un seul accès, à Almonte. En effet, cette municipalité a manifesté sa réticence à autoriser la construction de pontons dans l'estuaire du Guadalquivir, sur son territoire communal, pour l'amarrage des bateaux destinés aux visites ; elle a engagé des poursuites afin d'éviter ces équipements.

11. L'évolution du nombre de participants au pèlerinage du Rocío a été spectaculaire, depuis les six mille pèlerins qui le fréquentaient au milieu du XIX ${ }^{\mathrm{e}}$ siècle jusqu'au million et demi qui fut comptabilisé en 1988. L'augmentation de romeros s'est accélérée depuis la fin des années soixante et surtout dans la seconde moitié des années soixantedix. En 1980 on atteint le chiffre de un million de participants. Depuis, l'augmentation est plus lente et est arrivée à se stabiliser dans les dernières éditions du pèlerinage. 12. Paroles du Duc de Medina Sidonia, en 1773, citées par Granados (1989, p. 231). 13. Dans une lettre du 11 août 1834 adressée au magistrat Juez de la Villa, certains habitants d'Almonte, installés temporairement dans le hameau devant la menace d'une épidémie, fournissent une connaissance de l'état d'abandon total des résidents et de l'absence du moindre service, de sécurité notamment (Alvarez Gaston, 1978, pp. 103-104). En 1883, selon le constat fait dans un mémoire des Archives municipales d'Almonte, on comptait sept enfants d'âge scolaire, c'est-à-dire âgés de six à neuf ans, comme le prévoyait la Loi Moyano de 1857 alors en vigueur.

14. Acte Capitulaire du 1er mars 1976 et Rapport annuel du secrétariat de mairie, 1958, legs 304, fol. n¹ (Archives Municipales d'Almonte). Dans le rapport cité, il est dit littéralement : "Cette corporation a investi un important montant dans la planification d'un secteur adéquat, face à l'Ermitage, où autrefois arrivaient les eaux des marais. On a gagné avec ces opérations une grande bande de terrains sur les marais, qui donne de la place aux milliers de pèlerins qui se concentrent chaque année vers la Pentecôte ». 15. A cette date, la nouvelle corporation avait accompli sa première année de pouvoir. Ces données furent communiquées par écrit et verbalement au maire d'Almonte par quelques instituteurs du hameau, parmi lesquels se trouvait l'auteur de ces lignes. 16. Nous pûmes vérifier ces doutes sur le nom qu'il convenait de donner aux habitants habituels du village lors d'une conversation avec Madame Josefa María Pérez Domínguez, domiciliée dans la rue Bellavista. Elle nous confia qu'ils ne pouvaient plus s'appeler « Rocieros », puisque ce nom est donné à tous ceux qui se sentent liés au pèlerinage et aux célébrations du Rocío. Elle choisit l'expression de " gens du Rocío » pour éviter les confusions et identifier les autochtones du village, les différenciant également de ceux qui viennent d'autres communes et se sont installés récemment. Bien qu'il y ait eu des précédents, y compris au siècle dernier, cet effort d'autoidentification des habitants constitue une nouveauté frappante et un signe supplémentaire de changement culturel, conséquence de la conversion du Rocío en point d'attraction pour des individus et des institutions étrangères, avec une amplitude chaque jour croissante.

17. Ces données démographiques constituent un petit échantillon d'un travail que nous venons d'élaborer dans le cadre d'un programme plus important sur le Rocío, le 
professeur M.D. Murphy de l'Université d'Alabama (USA) et moi-même. Le traitement informatique des données est dû au professeur J. Bindon, de la même Université, que nous remercions pour cette aide précieuse.

18. Ces difficultés sont croissantes. Certains habitants du village viennent d'engager la construction d'un groupe d'habitations sociales, subventionnées par l'État, dans des terrains achetés à bas prix à la municipalité. Ces maisons, de dimensions et d'aspect identiques, se situeront dans la périphérie, formant un petit quartier. Elles offriront, assurément, un clair contraste avec les habitations traditionnelles, beaucoup plus grandes et variées, la majorité étant inoccupées pendant presque toute l'année. Dans aucun des plans urbanistiques élaborés jusqu'ici n'avait été envisagée cette revendication des habitants due à la pression démographique et à l'élévation du prix du terrain.

19. Santero est le nom donné au gardien de l'ermitage. Il assure occasionnellement les tâches liées à l'entretien interne et externe du temple et participe parfois aux célébrations du culte. Le santero vit normalement dans le village.

20. En 1993 le premier hôtel de l'histoire du village a été inauguré - il existait auparavant seulement deux pensions familiales - avec un nom très lié aux célébrations du Rocío : «Puente del Rey ». Une partie de ses annexes a été vendue en petits appartements à des particuliers. Ce type de « résidence ", si urbaine et fréquente dans la zone touristique de la plage, était jusqu'alors complètement ignorée du Rocío. 21. L'une des images qui illustrent au mieux cette discontinuité progressive et déjà visible et cette séparation entre le village et les marais proches est le « Paseo marismeño »; construit il y a quelques années, il vint se substituer en partie à l'ancienne clôture de pieux (piquets de bois) et de fils de fer barbelés et à la bordure sableuse et arborée contiguë. A sa place on peut voir aujourd'hui une promenade carrelée, des bancs métalliques et des plates-bandes de fleurs ornementales. Une erreur a fait que dans un document municipal, cette promenade a été dénommée «Paseo marítimo » [promenade maritime, N.D.T.], homonyme de celle qui avait été dessinée dans l'urbanisation touristique de la plage de Matalascañas.

22. «L'historien Ibn Hayyan affirme que dans al-Mama'in, c'est-à-dire dans les marais, on vit jusqu'à 3000 juments de reproduction et 100 étalons ", selon une étude intéressante sur la Zoohistoire d'Espagne (1991) d'Enrique de Obregón.

23. Société d'encouragement et de surveillance pour l'élevage. Voir dans ce numéro l'article de Vicente Jurado Doña (NDLR).

24. «On sait qu'Almonte détient une richesse considérable dans son élevage et que celle qui consiste à élever des juments, des chevaux et des porcs s'effectue presque toute l'année dans les marais de son territoire communal et que dans ces circonstances tout y est sauvage et indompté, car on ne peut rien enfermer dans des étables... »C'est avec ces considérations, entre autres, que vingt-six éleveurs de la Villa se présentent à la mairie en juin 1853, réclamant que leur bétail des marais puisse pâturer dans les oliveraies pendant l'époque estivale, à cause de la pénurie de nourriture dans les marais. Cf. Alvarez Gaston, R. (1978), op. cit. , p. 52.

25. Dans les premiers jours de janvier 1993, des affrontements graves se déroulèrent dans les marais entre les autorités de la protection de la nature et les éleveurs, en raison de la rareté des pâturages causée par une sécheresse prolongée. D'une certaine manière, ils cristallisaient une longue accumulation de désaccords et de tensions. Ce n'était pas, en tout cas, la première fois que ce type de conflit était apparu dans des 
circonstances similaires, bien que leur virulence et leur ampleur fussent toujours moins importantes.

26. De yegua, jument [N.D.T.]

27. Expression locale désignant l'opération qui consiste à rassembler et récupérer le troupeau des juments [NDT].

28. Ce terme provient du verbe «tondre » [atusar en espagnol et non atuzar, N.D.T.]. Nous l'avons transcrit tel qu'il se prononce dans cette zone, avec son zézaiement caractéristique.

29. Association des Éleveurs de Bétail des Marais.

30. En 1992 s'est constitué le «Club Hippique du Rocío », en grande partie dû à

l'initiative de la direction de l'Association des Éleveurs des Marais, concomitante à la déclaration municipale instaurant le Rocío « Village International du Cheval ». Ce Club a reçu des terrains municipaux pour construire un siège social où se célèbrent les nombreuses activités sportives organisées désormais régulièrement.

31. Cette enquête est l'un des instruments utilisés dans une étude socio-éducative encore inachevée à laquelle a collaboré le professeur D. Diego Ramírez. Son objectif va plus loin que le diagnostic sommaire que nous brossons ici. Il prétend finalement apporter des orientations susceptibles d'aider à l'amélioration de la présence de Doñana comme moyen éducatif des écoles de son environnement.

32. Coto : réserve de chasse [N.D.T.]

33. Les items de l'échantillon ont été sélectionnés à partir du manuel : González Faraco, J.C. (1988), Encuentros con Doñana. Programa de Educación Ambiental, Huelva, Coop. Marismas del Rocío, et des programmes d'enseignement que plusieurs professeurs ont développés dans le cadre du cycle supérieur de l'enseignement primaire à partir de ce manuel.

34. Cette déficience est soulignée par la perte du riche vocabulaire local d'espèces, outils, constructions, instruments traditionnels, etc.

35. Nous nous référons aux prévisions du fameux Rapport sur les stratégies pour le développement socio-économique durable de la périphérie de Doñana, Séville, 1992, réalisé par la Commission internationale d'experts sur le développement de Doñana, nommée par le Président du gouvernement d'Andalousie.

\section{RÉSUMÉS}

L'auteur décrit et interprète les effets produits par la coïncidence, en une même région, d'un important espace naturel protégé (le Parc national de Doñana), et de processus de développement économique rapides autour de cet espace. Une telle situation provoque des conflits et des contradictions dans les communautés locales. L'analyse des usages économiques traditionnels, des rituels festifs et des processus de socialisation des nouvelles générations, à partir d'une approche ethno-sociologique, permet d'établir quelques unes des antinomies culturelles, manifestes ou latentes, issues de cette situation duale, peu intégrée, tant au plan territorial que social ou économique.

Doñana : tradition and social change. Paradoxes in a charismatic natural space. 
The Doñana National Park Region being divided into, on the one part, a large preserved natural space and, on the other part, surroundings areas experiencing fast economic developments, conflict situations have happened to raise within local communities. According to an ethnosociological approach, ritual social uses, socialization of young generations and economic habits are analyzed to point out some cultural antinomies, either patent or latent, expressing the dual territorial situation.

\section{Doñana : tradición y cambio social. Antinomias en un espacio natural carismático.}

Este artículo describe e interpreta los efectos producidos por la coincidencia, en un mismo territorio, de un importante espacio natural protegido y unos rápidos procesos de desarollo económico en su entorno. Se trata de analizar, principalmente, los conflictos o contradicciones culturales que tal situación provoca en las comunidades locales. Con tres temas que se refieren a usos económicos tradicionales, rituales festivos y procesos de socialización de las nuevas generaciones, y usando de una óptica etnosociológica, el autor establece algunas de las antinomias culturales, manifiestas o latentes, que nacen de esta situación dual y poco integrada, en lo territorial, en lo social y en lo económico.

\section{INDEX}

Mots-clés : Espagne, Représentations de la nature, Espaces protégés, Pratiques de la nature, Pratiques festives, Andalousie

Palabras claves : España, Representaciones de la naturaleza, Espacios protegidos, Prácticas de la naturaleza, Prácticas festivas, Andalucia

Keywords : Spain, Representations of nature, Protected areas, Practices of nature, Festival practices, Andalousia

\section{AUTEUR}

\section{JUAN CARLOS GONZÁLEZ FARACO}

Professeur à l'Université de Huelva, il est spécialiste des questions d'éducation relatives à l'environnement. Il poursuit depuis des années des recherches ethno-sociologiques et pédagogiques relatives à la conservation de la nature, dont certaines portent sur les communautés situées dans des espaces naturels protégés, tel que le Parc national de Doñana. 